\title{
Das Erkenntnisobjekt der Sozialgeographie aus handlungstheoretischer Sicht
}

\section{Das Unbehagen an der gegenwärtigen Forschungspraxis}

Die etablierten Schulen und Ansätze der Sozialgeographie werden seit einiger Zeit einer dezidierten Kritik unterzogen, die auf der Basis verschiedenartiger erkenntnistheoretischer Positionen vorgetragen wird und mit sehr unterschiedlichen (aber auch unterschiedlich plausiblen) Argumentationen operiert, jedoch grundsätzliche Übereinstimmung in der Ablehnung der gegenwärtigen Forschungspraxis aufweist.

Von dieser Ablehnung war zunächst die «Wien/Münchener Schule der Sozialgeographie» betroffen, der vor allem der mangelnde Bezug zur sozialwissenschaftlichen Theoriebildung vorgeworfen wird (vgl. z. B. E. WIRTH, 1977). Aber auch die Arbeitsrichtungen der choristisch-chorologischen Sozialraumforschung und der verhaltens- und wahrnehmungswissenschaftlichen Geographie gerieten zunehmend in das Kreuzfeuer einer Kritik, die nicht bloß Einzelaspekte oder strittige Detailfragen berührt, sondern die Fundamente und Kernbereiche der betreffenden Paradigmen in Frage stellt. Die «antiszientistische» Verurteilung des "spatial approach» ist natürlich vor dem Hintergrund der Abrechnung mit dem Neopositivismus und dem Kritischen Rationalismus und deren nobler Zurückhaltung gegenüber Sinn- und Werturteilsfragen zu sehen (vgl. z. B. P. SEDLACEK, Hrsg., 1982).

Die antiszientistische Stoßrichtung betrifft auch die verhaltenswissenschaftlich orientierte Humangeographie, die selbst in ihren neueren und «organismisch» konzipierten Varianten das Erbe des Behaviorismus und das grundlegende Modell der ReizReaktions-Mechanismen nicht leugnen kann. Die hauptsächlich artikulierten Gegenpositionen beziehen sich auf (vielfach marxistisch orientierte) gesellschaftstheoretische Entwürfe einer präskriptiven und «eingreifenden» Sozialwissenschaft, auf phänomenologische und existentialphilosophisch fundierte Ansätze einer «humanistischen Geographie» oder auf Vorschläge einer handlungstheoretischen Neukonzeption, die sich meist auf M. WEBER, A. SCHÜTZ und T. PARSONS sowie auf die Ethnomethodologie stützen. Einigkeit zwischen diesen drei Hauptlinien der Kritik besteht vor allem in der Auffassung, daß menschliches «Tun» (und natürlich auch menschliches Tun im Raum) keinesfalls als «Verhalten» dargestellt und mit verhaltenswissenschaftlichen Modellen beschrieben werden könne. Für eine angemessene Erklärung sei es vielmehr notwendig, menschliches Agieren in seiner Intentionalität und Reflexivität zu erfassen, seine Eingebundenheit in normative Strukturen und Wertesysteme sowie seine symbolischen, affektiven und emotionalen Komponenten zu berücksichtigen.

Im folgenden sollen einige Überlegungen zum Vorschlag einer handlungstheoretischen Neukonzeption der Sozialgeographie (E. WIRTH, 1981, P. SEDLACEK, 1982, B. WERLEN, 1983 und 1986) angestellt werden, wobei neben Konsequenzen für das Erkenntnisobjekt dieses Faches auch meß- und erhebungstechnische Gesichtspunkte einer Realisierung derartiger Ansätze zu bedenken sind.

\section{Ein handlungstheoretisches Modell der Mensch-Umwelt-Interaktion}

Als Ausgangspunkt für die folgenden Überlegungen soll ein einfaches Modell der Mensch-UmweltInteraktion dienen, mit dessen Hilfe einige der in den Handlungstheorien als relevant angesehenen Grundbegriffe und Beziehungszusammenhänge angedeutet werden. Die Darstellung der Abb. 1 bezieht sich noch nicht auf das (eingeschränktere) Erkenntnisobjekt der Sozialgeographie, sondern auf den übergeordneten Problematisierungsstil, der im Rahmen einer humanökologischen Betrachtungsweise angemessen erscheint.

Im Gegensatz zu den Stimulus-Response-Konzeptionen des verhaltenswissenschaftlichen Paradigmas, in denen der Mensch quasi als Automat angesehen wird, also als Wesen, das auf äußere Anstöße und Rahmenbedingungen gemä $\beta$ eines angeborenen oder erlernten "Programms» reagiert, gehen handlungstheoretische Überlegungen vom Primat subjektiver, gruppen- und kulturspezifischer Sinnund Zielsetzung aus, die es individuellen und kollek-

Peter Weichhart, Univ. Doz., Dr., Institut für Geographie der Universität Salzburg, Akademiestr. 20, A-5020 Salzburg 


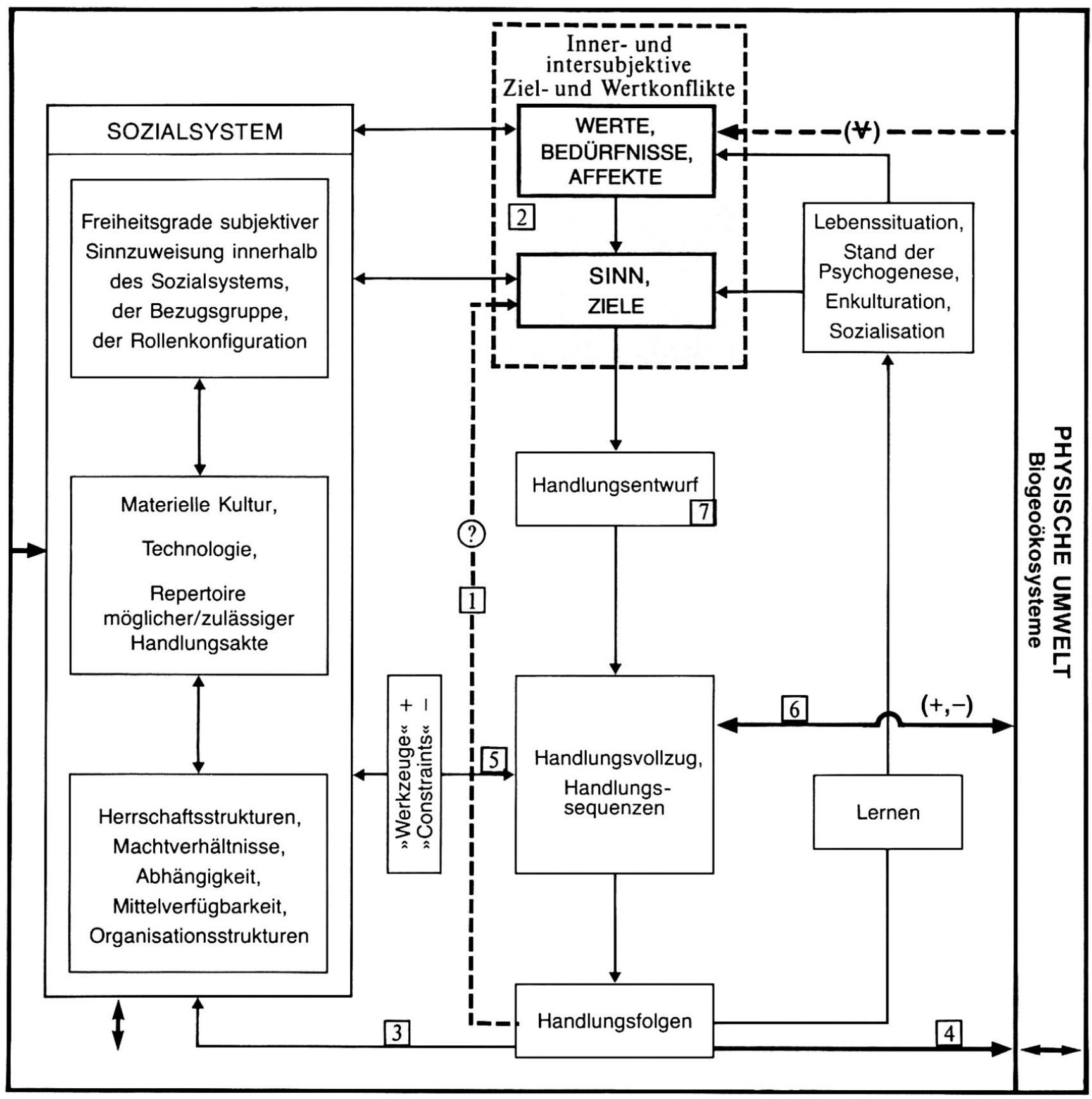

Abb.1: Ein handlungstheoretisches Modell der Mensch-Umwelt-Interaktion

tiven Handlungsträgern erlaubt, innerhalb spezifischer Grenzbedingungen system- und interaktionssteuernde Größen weitgehend autonom selbst zu generieren und zu variieren. Mensch-Umwelt-Beziehungen werden nach dieser Anschauung also als teleogenische Systeme verstanden (vgl. A. LOCKER und N.A. COULTER, Jr., 1977 und P. WEICHHART, 1986a, Abb. 5), deren Steuergrößen durch systemimmanente Prozesse erst produziert werden. (Mit «Umwelt» ist hier natürlich der Gesamtbereich menschlicher Interaktionspartner und -objekte gemeint, der vom Aspekt der physischen Umwelt über den personalen, sozialen, kulturellen bis zum ideo- logischen Umweltaspekt reicht). Gleichzeitig implizieren handlungstheoretische Ansätze die Interpretation von Mensch-Umwelt-Beziehungen als selbstreferentielle Systeme, deren Elemente und Relationen sich durch den Rückbezug auf die eigenen Strukturen und Operationen konstituieren (vgl. N. LUHMANN, 1985).

Bezogen auf den in Abb. 1 angesprochenen einfachsten Fall eines Individuums, kann jedes menschliche Tun (aber auch jede Unterlassung eines Tuns), das von diesem Akteur mit einem subjektiven Sinn verbunden wird, als «Handeln» bezeichnet werden. Auch «Sinn» ist als selbstreferentielles Phänomen 
zu begreifen, das Möglichkeiten des Erlebens und Handelns eröffnet, die Komplexität der Welt für psychische und soziale Operationen handhabbar macht und damit als Bezugsraster und Verweisstruktur von Intentionalität fungiert (vgl. ebda., Kapitel 2). Damit soll angedeutet werden, daß Sinngehalte und darauf bezogene Handlungen nicht in einfacher linearer Weise zusammenhängen, sondern daß Sinn im Handlungsvollzug auch neu entworfen oder variiert und vielfach sogar erst konstituiert wird. Inhaltlich faßbar und inner- bzw. intersubjektiv artikulierbar werden Sinnstrukturen in der konkreten Ausprägungsform von Handlungszielen. Sinn und Ziele stehen in Beziehung mit normativen und präskriptiven Bedeutungszusammenhängen, die als Werte, Bedürfnisse, Postulate, Affekte und Symbole dem Handlungsträger verfügbar sind, innerhalb gewisser Grenzen der subjektiven Argumentation zugänglich gemacht werden können und zumindest im Rahmen spezifischer Lebenssituationen den Charakter bejahter Gewißheit besitzen. Sie sind Bestandteil und normative Referenzgrößen der kognitiven Repräsentation der Wirklichkeit im Bewußtsein menschlicher Individuen.

Die Darstellung menschlichen Tuns als «Handeln» wird dann möglich, wenn die Reflexivität und Intentionalität des Akteurs ausdrücklich berücksichtigt wird und dieses Tun als bewußt erwogener, nicht determinierter, absichtlich auf ein Ziel hin entworfener Akt beschrieben werden kann (B. WERLEN, 1986, S. 5). In funktionalistischer Redeweise könnte man auch sagen, daß das Handeln eines Akteurs von ihm selbst durch die eigenen Wert- und Sinnkonfigurationen gesteuert wird. Wesentlich ist dabei die Fähigkeit zur Antizipation des Handlungsziels und seiner zweckhaften Konkretisierung sowie zur Abschätzung der erwarteten Handlungsfolgen und der Angemessenheit der gewählten Mittel.

In vielen Fällen läuft menschliches Tun allerdings gewohnheitsgemäß und gleichsam automatisch ab. Auch habitualisiertes und nicht aktuell argumentationsvorbereitetes Tun, das als Quasi-Verhalten bezeichnet wird (P. SEDLACEK, 1982, S. 195), kann aber bei entsprechendem Anstoß vom Akteur nachträglich auf einen Sinn bezogen und in Zusammenhang mit seiner Zielkonfiguration dargestellt werden. Der Handlungsträger steht also (und nicht nur im Falle des Quasi-Verhaltens) häufig in einer ähnlichen Situation wie der Sozialwissenschaftler: Er ist genötigt, sein eigenes Tun zu interpretieren, es sich selbst plausibel zu machen, mit den eigenen Zielen in Einklang zu bringen und es damit sich selbst gleichsam als Konsequenz der bejahten Sinngehalte zu «erklären».

In analytischer Hinsicht wird der sequentielle Charakter von Handlungen meist durch drei Prozeßeinheiten angenähert (vgl. z. B. B. WERLEN, 1986, S. 5/ 6):
- der Handlungsentwurf als vorbereitende Reflexion und Planung konkreten Tuns, in der ZweckMittel-Relationen überlegt sowie spezifische Mittel ausgewählt werden, antizipierend die erwarteten Konsequenzen der Zielrealisierung zu bedenken sind und die Rechtfertigung der angestrebten Ziele und Realisierungsverfahren vor dem Hintergrund der als gültig akzeptierten Werte stattfindet;

- der Handlungsvollzug, der (auch in einfachen Fällen) meist als Sequenz einzelner Handlungsschritte zu sehen ist, bei dem unterschiedliche Mittel oder «Werkzeuge» eingesetzt werden;

- die Handlungsfolgen, die als tatsächliches oder vermeintliches Resultat der durchgeführten Einzelschritte des Handlungsvollzugs Auswirkungen auf materielle oder immaterielle Systemzustände besitzen.

Klammert man pathologische Grenzfälle (Autismus) und scheinbar sinnloses Handeln aus (das seinen «Sinn» etwa erst in einer psychoanalytischen Darstellung offenbart oder durch den Bezug der persönlichen Sinnkrise charakterisiert ist), dann muß jedes menschliche Handeln im Kontext des umgebenden sozialen Systems interpretiert werden, durch das die Rahmenbedingungen für die drei angeführten Prozeßeinheiten festgelegt werden. Subjektive Handlungsziele, Werte und Sinnzusammenhänge sind begrenzt auf das Repertoire der im jeweiligen Sozial- und Kultursystem zulässigen bzw. verfügbaren normativen und präskriptiven Strukturen. Die Spielräume individueller Sinnfindung werden eingeengt durch die Freiheitsgrade und tolerierten Variationsmöglichkeiten, die im betreffenden Sozialsystem erlaubt sind, wobei Bezugsgruppe und spezifische Rollenkonfiguration des jeweiligen Akteurs sicher eine wichtige Rolle spielen. Von diesen Variationsmöglichkeiten auf der Werteebene, die durch die bestehenden Machtverhältnisse vorgeformt werden, sind auch die zeitlichen Veränderungen und Evolutionsmöglichkeiten des gesamten Sozialsystems bestimmt (Wertwandel).

Der konkrete Vollzug einzelner Handlungsschritte greift auf die verfügbaren und zulässigen Anwendungsmöglichkeiten und die Gegebenheiten der materiellen Kultur (wozu auch die räumlichen Infrastrukturpotentiale zu rechnen sind) und derTechnologie zurück, die gleichsam als Werkzeuge genutzt werden können (in der Abbildung mit [5] gekennzeichnet). Er ist in sehr direkter Weise von der Mittelverfügbarkeit des Handlungsträgers und vor allem von den bestehenden Macht- und Herrschaftsverhältnissen abhängig, die als Zwänge erlebt werden und die objektiven Grenzen möglicher Handlungsvollzüge determinieren. Als Werkzeuge oder Constraints können auch die Gegebenheiten der physischen Umwelt wirken 6, in die das Sozialsystem eingebettet ist und auf deren Grundlage die materielle Kultur basiert. 
Handlungen haben (gewollte und ungewollte) Folgen, die auf das Sozialsystem 3 und die physische Umwelt 4 rückwirken. Im Erleben der Handlungsfolgen und der Bestrafung oder Belohnung durch die soziale Umgebung findet die Eingliederung des Akteurs in das Kultursystem statt, lernt er die Spielräume und Grenzen seiner Handlungsmöglichkeiten kennen. Subjektive Sinnfindung gewinnt durch derartige Lernvorgänge über den Prozeß der Sozialisation ihren Standort im übergeordneten sozialen Gefüge, wobei durch Persönlichkeitsmerkmale und vor allem durch spezifische Lebenssituationen Entwicklungslinien vorgezeichnet und Akzente gesetzt werden.

Durch die Summe aller Handlungsfolgen werden materielle und immaterielle Zustände des übergeordneten Gesamtsystems (also auch räumliche Infrastrukturpotentiale, die räumliche Konfiguration sozialer Beziehungen oder Landnutzungssysteme) beeinflußt, verändert oder gar erst erzeugt.

Handlungsfolgen werden vom Akteur mit den selbst vorgegebenen Zielen verglichen. Der «Erfolg» eines Tuns ist dann gegeben, wenn durch den Handlungsvollzug die Intentionen realisiert werden konnten, Systemzustände bewirkt wurden, die mit der Sinnstruktur korrespondieren. Dieser Rückkoppelungsproze $B$ ist für das Verständnis menschlicher Handlungen und für humanteleologische Erklärungsmodelle überaus bedeutsam. Der Mensch besitzt eine nahezu unglaubliche Kapazität, diesen Vergleich zwischen ursprünglichen Intentionen und den tatsächlich bewirkten Handlungsfolgen $\square$ in Abb. 1) durchzuführen und zu interpretieren. Dabei handelt es sich um argumentative Prozesse der aktiven Umdeutung, durch die Diskrepanzen zwischen Intention und Handlungsergebnis abgebaut oder zum Verschwinden gebracht werden. Anders formuliert: Der Mensch verfügt über ein sehr großes Repertoire räsonierender Bewußtseinsakte, durch die nahezu beliebige Zusammenhänge zwischen Sinnstruktur und Handlungsfolgen hergestellt werden können, die subjektiv (aber auch gruppen- oder gar kulturspezifisch) als "wahr», "richtig», «gültig» oder «angemessen» erscheinen (vgl. dazu z. B. das bei H. MEHAN und H. WOOD, 1979, S. 29-34 diskutierte Beispiel).

Ein erster möglicher Effekt dieser Rückkoppelung bei Korrespondenz zwischen Resultat und Ziel einer Handlung kann darin bestehen, daß durch die erfolgreiche Realisierung von Zielen neue Ziele generiert werden. Verschiedene Ergebnisse der Bedürfnistheorie lassen die Hypothese plausibel erscheinen, daß mit der Verwirklichung niederrangiger Bedürfnisse Appetenz nach höherrangigen Bedürfnissen (Wachstumsmotive wie Selbstverwirklichung) geweckt wird, was zu neuen Handlungssequenzen führt.

Sehr häufig ergibt der Vergleich aber keine oder eine schlechte Übereinstimmung zwischen Absicht und Folgen der Handlung. Dann stehen dem Handlungsträger verschiedene Strategien zur Lösung dieser Spannung zur Verfügung. Neben der Wiederholung der erfolglosen Handlungssequenz mit verstärkter Anstrengung und dem Einsatz anderer Mittel sind hier vor allem argumentative und interpretative Bewußtseinsprozesse von Bedeutung. Spannungslösung kann etwa dadurch erreicht werden, daß eine «Schuldzuweisung» für das Scheitern an die Rahmenbedingungen und Interaktionspartner erfolgt. Eine weitere «Problemlösung» besteht in der Uminterpretation der Folgen: Negative Aspekte der Handlungssequenz werden ignoriert, beiseite gestellt oder verdrängt, positive werden überbetont. Damit stimmen in der subjektiven Weltsicht des Akteurs die Folgen seines Tuns plötzlich doch mit den Zielen überein. Eine weitere Strategie besteht in der nachträglichen Umdeutung der subjektiven Sinnstrukturen. Wenn der Akteur die erlebte Realität nicht im Sinne der gesetzten Ziele verändern kann, dann werden eben die Werte an die Realität angepaßt und die getroffenen Entscheidungen und Handlungsvollzüge nachträglich rationalisiert. Derartige psychische Umdeutungsprozesse, die durch unzählige Untersuchungen im Rahmen verschiedener Konsistenztheorien der Psychologie (etwa der Theorie der kognitiven Dissonanz) sehr gut bestätigt sind, werden dem Akteur aber gar nicht bewußt, sie werden von ihm keineswegs als «Ausrede» oder «Ausflucht» empfunden, sondern erscheinen subjektiv als völlig schlüssig, rational und logisch. Aus der «objektiven Sicht» eines externen Beobachters muß die gleiche Interpretation oft als völlig unsinnig erscheinen, sie wird als irrational oder a-rational klassifiziert, weil sie der Prüfinstanz der klassischen Logik nicht standhält. Ein solches Urteil ist aber zweifellos nicht zutreffend, denn die subjektive Deutung der Wirklichkeit stellt für den Handlungsträger ja räsonierendes, vernünftiges Überlegen dar, sie hat für ihn eine argumentative Struktur. Es scheint angemessen, hier von subjektiver Rationalität und subjektiver Logik zu sprechen. Daß diese Art subjektiv-rationaler Weltsicht keineswegs auf Individuen mit begrenzter Handlungs- oder gar Denkkompetenz beschränkt, sondern sicher auch für Entscheidungsträger in höchsten politischen, wirtschaftlichen und sozialen Funktionen charakteristisch ist, können Beispiele aus aktuellen tagespolitischen Diskussionen (aber $z$. B. auch viele Rechtfertigungsversuche und «Begründungen" raumordnungspolitischer und raumplanerischer Entscheidungen) allenthalben augenscheinlich demonstrieren. Derartige Umdeutungsprozesse werden keineswegs nur von Individuen durchgeführt, sie sind als gängige und allgemein akzeptierte Denkmuster auch Bestandteile gruppenund kulturspezifischerWeltsicht.

Mit ähnlichen Denkmechanismen ist es dem Menschen auf einfache Weise und äußerst wirksam mög- 
lich, obsolete, unehrenhafte, egoistische oder ethisch unhaltbare Einzelziele so umzuinterpretieren, daß sie in einem direkten Deduktionszusammenhang mit allgemein akzeptierten hohen und höchsten Werten gebracht werden können. Die Geschichte der Menschheit und das aktuelle Weltgeschehen bieten genügend Belege dafür, welche grauenhaften Handlungen durch den Rekurs auf anerkannte Werte wie Ehre, Treue, Freiheit, Religion ... subjektiv und gruppenspezifisch gerechtfertigt werden, wobei die (oft nur durch wilde mentale Verrenkungen mögliche) «Argumentation» den betreffenden Handlungssubjektiven durchaus korrekt, gültig, stimmig, zwingend und konsistent erscheint.

Ähnliche Denkmuster einer subjektiven Rationalität werden eingesetzt, wenn es um die Bewältigung inner- und intersubjektiver Ziel- und Wertkonflikte geht [2] in Abb. 1) Auch hier dienen subjektive und gruppenspezifische (ideologische) Abbau-, Verdrängungs- und Harmonisierungsstrategien als Mittel der inneren Überzeugung, die letztlich zum Ergebnis einer Spannungs- oder Problembewältigung dadurch führen, daß die Konflikte verdeckt, mit Überzeugung geleugnet oder als nicht existent angesehen werden. Bei intersubjektiven Zielkonflikten findet eine Austragung meist über Macht- und $\mathrm{Ab}$ hängigkeitsstrukturen statt, wobei sich natürlich solche subjektiven oder gruppenspezifischen $\mathrm{Mu}$ ster der Weltdeutung als besonders «praktisch» erweisen, mit deren Hilfe eine Rechtfertigung oder Legitimierung eigener Machtansprüche durch im jeweiligen Gesellschaftssystem gültige hochrangige Werte möglich ist (vgl. z. B. den Begriff des «Gotteslehens» in der mittelalterlichen Feudalgesellschaft).

Es kann angenommen werden, da $\beta$ die Summe der Folgen und Konsequenzen der Handlungen aller individuellen und kollektiven Akteure, die in einem bestimmten Ausschnitt der Erdoberfläche wirksam sind, dessen kultur- und sozialräumliche Strukturen entstehen ließen und aktuell umformen. Sollen diese Strukturen und Prozesse im Raum auf mehr als oberflächliche Weise erklärt werden, dann ist es notwendig, mit Hilfe humanteleologischer Ansätze die dahinterstehenden oder bewirkenden Handlungsabläufe zu rekonstruieren, wobei also bis auf die Ebene der eigentlichen Steuergrößen, nämlich der Wert- und Sinnzusammenhänge, zurückgegriffen werden muß.

Wenn man das hier in aller Kürze diskutierte handlungstheoretische Modell als erste Annäherung einer adäquaten Darstellung der Gegebenheiten von Mensch-Umwelt-Interaktionen akzeptiert, dann erscheint es möglich und sinnvoll, dieses Modell auch für die eingeengteren Problem- und Fragestellungen der Sozialgeographie als forschungsleitende Perspektive anzuwenden.

\section{Folgerungen für das Erkenntnisobjekt der Sozialgeographie}

Obwohl es zurzeit sehr unterschiedliche und miteinander konkurrierende Versuche gibt, das Erkenntnisobjekt der Sozialgeographie zu formulieren, dürfte sich zwischen den Vertretern der verschiedenen Schulen und Arbeitsrichtungen wohl ein Konsens darüber erzielen lassen, da $\beta$ die spezifische Weltsicht dieser Teildisziplin in der Beschäftigung mit sozialen Phänomenen im Raum, in der Erklärung der erdräumlichen Differenzierung der «sozialen Welt» und ihrer materiellen Ausprägungsformen besteht. Wenn man nun versucht, diese (sehr vage formulierte) Aufgabenstellung im Rahmen handlungstheoretischer Konzeptionen zu realisieren, dann muß der übliche Kanon konkreter Forschungsaufgaben um eine ganze Reihe bisher kaum beachteter Fragen erweitert werden. Besonders dringlich erscheint dabei die Befassung mit den in der folgenden (keineswegs vollständigen) Aufzählung angeführten Problemkreisen:

1. An erster Stelle muß die Beschäftigung mit den Phänomenen «Wert» und «Sinn» genannt werden, die bisher in der Forschungspraxis der Humangeographie auf allen Maßstabsebenen der Analyse völlig unzureichend oder gar nicht thematisiert wurden. Es wird notwendig sein, Werte nicht nur als unabhängige, sondern auch als abhängige Variablen zu betrachten, also auch auf die Genese von Werten einzugehen. Eine Auseinandersetzung mit der interdisziplinären Wertforschung und der philosophischen Ethik läßt sich demnach kaum vermeiden. Als Beispiel für einen konkreten Schritt in diese Richtung kann etwa die Dissertation von G. BÄUERLE (1984) angeführt werden (vgl. z. B. auch K.-H. HILLMANN, 1981). Inwieweit die Sozialgeographie dabei von einer empirischen Analyse der bestehenden handlungswirksamen Wertesysteme zu einem aktiv-präskriptiven und damit «eingreifenden» Ansatz fortschreiten soll, wird wohl dem persönlichen Gewissen und der weltanschaulichen Position des einzelnen Wissenschaftlers überlassen bleiben müssen.

2. Als ähnlich bedeutsam für humanteleologische Erklärungsmodelle und für «hermeneutische Interpretationen» (vgl. z. B. G. HARD, 1985) muß der Problemkreis der «subjektiven Rationalität» eingeschätzt werden. Wenn inneres und äußeres Argumentieren des Menschen nicht den Gesetzen der klassischen Logik entspricht, und unzählige empirische Befunde aus dem Bereich der Attributionstheorie (vgl. z. B. W. HERKNER, Hrsg., 1980) belegen, da $B$ diese Annahme generell und auch für Handlungsträger mit höchster Kompetenz zutrifft, dann ist die Rekonstruktion der argumentationsleitenden subjektiven Logik eine unabdingbare Voraussetzung für das Verstehen von Handlungen. Die 
eigenartige Brüchigkeit und Inkonsequenz, die Ambivalenzen und die Logik des «entweder und oder", durch die lebensweltliche Phänomene charakterisiert sind (vgl. D. REICHERT, 1985), können nur durch die Abkehr von jenem «dogmatischen Logizismus» (G. H. v. WRIGHT. 1972, S. 149), der auch das gegenwärtige Menschenbild der Geographie sowohl in der «homo oeconomicus-» als auch in der «satisfizer-Variante» prägt, eine angemessene wissenschaftliche Darstellung finden. Besonderes Augenmerk ist auch im Kontext raumrelevanter Entscheidungsprozesse den Argumentations- und Legitimationsmustern jener individuellen und institutionellen "Gatekeeper» zu widmen, die an den Schlüsselpositionen gesellschaftlicher Systeme Kontrollund Steuerfunktionen innehaben.

3. Damit in Zusammenhang steht die Notwendigkeit, im Rahmen sozialgeographischer Arbeit die subjektiven und gruppenspezifischen Dimensionen menschlicher Weltsicht, also die Trivialontologie der Lebenswelt zu erfassen. Wir wissen sehr wenig darüber, welche Grundstrukturen die «Privatontologien» besitzen, nach denen Lebenswelt im Kontext des Alltagshandelns erfahren wird. Fest steht, daß das Beziehungs- und Ordnungsgefüge subjektiver (aber auch gruppen- und kulturspezifischer) Wirklichkeit als komplexes, vieldimensionales Konstrukt verstanden werden muß, dessen differenzierbare Einzelelemente und phänomenologischen Grundeinheiten wohl eher nicht den Kategorien neopositivistischer Sozialwissenschaft entsprechen. Aus dieser Erkenntnis resultieren das immer stärker werdende Interesse der Soziologen und Historiker an der «Alltagskultur» und die (oft etwas esoterisch anmutenden) Versuche der Ethnomethodologie, die als selbstverständlich hingenommenen Strukturen der Alltagswelt transparent zu machen. In der Geographie kann eine Entsprechung in den Programmen der «humanistischen» Ansätze gefunden werde, die auch als Reaktion auf jene Auswüchse technokratischer Überheblichkeit des positivistischen Wissenschaftsbetriebes zu sehen sind, gegen die T. HÄGERSTRANDs (1970) provozierende Frage gerichtet war, wo denn die Menschen in der Raumforschung blieben. Als Beispiel für empirische Forschungsfelder, in deren Rahmen versucht wird, die vorgeblich praxisrelevante Weltsicht von Experten auf jene der Betroffenen zu relativieren, können Bereiche der mikroanalytischen Wanderungstheorie (vgl. z. B. P. WeICHHART, 1986b) und die interdisziplinären Anstrengungen zum Thema «Lebensqualität» angeführt werden (vgl. z. B. M. PACIONE. 1982).

4. Im Rahmen handlungstheoretischer Konzeptionen wird es auch notwendig sein, die mit dem bisherigen Methodeninventar kaum faßbaren symbolischen und emotionalen Komponenten von Raumwahrnehmung und Raumbewertung dezidiert zu berücksichtigen. Als zukunftsweisender Einstieg in diese Thematik können jüngste Aktivitäten der deutschsprachigen Humangeographie zum Problemkreis «regionale Identität» angesehen werden.

5. Notwendig erscheint auch eine systematische Aufarbeitung des in bestimmten sozialen Systemen verfügbaren Repertoires möglicher oder zulässiger Handlungsakte und die Mitarbeit an den Versuchen, Typologien von Handlungen und Handlungselementen zu erstellen. Für die Sozialgeographie wäre hier vor allem die Auseinandersetzung mit dem räumlichen Kontext von Handlungen von Bedeutung.

6. Schließlich sei noch auf die Notwendigkeit verwiesen, bei der Analyse raumbezogener Handlungen und ihrer Folgen auf die differenzierende Wirkung der Persönlichkeitsstruktur individueller Akteure einzugehen, die ja die kleinste Analyseeinheit auf der Dimension des Handlungsträgers darstellen. Die eigenartige Reserviertheit der deutschen Sozialgeographie gegenüber dem Erkenntnisbereich «Individuum und Raum» wird von Fachvertretern des englischen Sprachraums nicht geteilt. Dort finden sich ausdrücklich programmatische Vorschläge zu einer Berücksichtigung persönlichkeitspsychologischer Theorien in geographischen Erklärungsmodellen (vgl. z. B. D. J. WALMSLEY. 1982). Die Arbeit von T. ABT(1983) demonstriert, daß sogar der Einsatz von Theorien der Tiefenpsychologie zum Verständnis sozialräumlicher Prozesse beitragen kann.

\section{Erhebungstechnische Konsequenzen}

Abschließend ist anzumerken, daß eine handlungstheoretische Orientierung auch schwerwiegende meß- und erhebungstechnische Konsequenzen nach sich zieht (vgl. z. B. P. WEICHHART, 1986b, Abschnitte 2.3 bis 2.5). Makroanalytische Ansätze, bei denen mit aggregierten Datenmengen operiert wird, sind meist nicht brauchbar, die üblichen Meß- und Analyseinstrumente sowie ein großer Teil der gängigen sozial- und raumwissenschaftlichen Indikatoren lassen sich nur begrenzt verwenden. Auch Überlegungen zur Bestimmung von Stichproben, zur Datenrepräsentativität und zum Thema der Signifikanzprüfungen erhalten hier besondere Akzente.

Dieser Ansatz geht ja davon aus, daß Handlungsvollzug als Aneignungsprinzip von Umwelt gelten muß und sich die Inhalte der Weltsicht und desTuns von Akteuren durch den Verweis auf die Handlungsziele erst konstituieren. Es muß daher jede Erhebung, in der empirische Daten losgelöst von konkreten Handlungsbezügen ermittelt werden, als völlig unangemessen erscheinen. Aus diesem Blickpunkt sind z. B. Wohnwunschstudien der mikroanalytischen Wanderungsforschung, die sich auf eine repräsentative Stichprobe der Gesamtbevölkerung 
(oder auf besonders «handliche» Probandengruppen wie Studenten) und nicht ausdrücklich auf Personen beziehen, die zum Zeitpunkt der Befragung tatsächlich auf Wohnungssuche sind, deren aktuelle Lebenssituation also mit den Inhalten der Erhebung korrespondiert, als unbefriedigend und wenig zielführend abzulehnen. Ähnlich sinnvoll wäre es, wollte man Personen ohne Führerschein nach den Kriterien und Urteilsdimensionen für einen Autokauf befragen.

Neben den individuellen und kollektiven Akteuren ist in handlungstheoretisch fundierten Untersuchungen daher eine weitere Grundeinheit der Analyse unabdingbar, nämlich jene des persönlichen (oder kollektiven) Projekts. Dieses Konzept wurde im Rahmen der Zeitgeographie und (unabhängig davon) in der neueren Persönlichkeits- und Umweltpsychologie entwickelt (B. R. LITTLE, 1983). Projekte werden als Handlungs- und Ereignisgruppen aufgefaßt, die aus Einzelaktivitäten, Teilzielen, Interaktionspartnern und Hilfsmitteln zusammengesetzt sind und zur Verwirklichung übergeordneter Ziele unternommen werden (vgl. T. CARLSTEIN, 1982, S. 47). In diesem Sinne können etwa Wanderungsprozesse als Projekte der betreffenden Haushalte aufgefaßt werden.

Wegen des Prozeßcharakters von Handlungen und der vielfältigen Rückkoppelungen durch argumentierende und attribuierende Reflexionen der Akteure müssen handlungstheoretische Untersuchungen wohl überwiegend als zeitliche Längsschnittanalysen angelegt werden. Panelstudien gängigen Zuschnitts sind dafür aber nicht ausreichend. Es wird vielmehr notwendig sein, eine handlungsbegleitende Datenaufnahme (teilnehmende Beobachtung, Tagebuchaufzeichnungen, Serien von Tiefeninterviews) zu bewerkstelligen und die im zeitlichen Ablauf der Handlungssequenzen stattfindenden Reflexionen, Urteile und Wertungen des Handlungsträgers im Zusammenhang zu rekonstruieren. Der methodische Aufwand für ein solches Vorgehen (vgl. z. B. P. WEICHHART, 1986b, Abschnitt 6.5) muß sicher sehr hoch veranschlagt werden. Er wird wohl das größte Hindernis für eine Verwirklichung handlungstheoretischer Konzepte in der empirischen Forschungspraxis der Sozialgeographie darstellen.

\section{Literatur:}

ABT, T. (1983): Fortschritt ohne Seelenverlust. Versuch einer ganzheitlichen Schau gesellschaftlicher Probleme am Beispiel des Wandels im ländlichen Raum. - Bern.

BÄUERLE, G. (1984): Der Freiraum als räumliches Wertobjekt. - München (= Minerva Publikation).

CARLSTEIN, T. (1982): Time Resources, Society and Ecology. On the Capacity for Human Interaction in Space and Time. Vol. 1: Preindustrial Societies. - London, Boston and Sydney (= Lund Studies in Geography, Ser. B., No. 49).
HARD, G. (1985): Städtische Rasen, hermeneutisch betrachtet - Ein Kapitel aus der Geschichte der Verleugnung der Stadt durch die Städter. - In: Festschrift zum 60. Geburtstag von Dr. Elisabeth Lichtenberger. - Klagenfurt (= Klagenfurter Geographische Schriften, H. 6), S. 29-52.

HERKNER, W. (Hrsg., 1980): Attribution - Psychologie der Kausalität. - Bern, Stuttgart und Wien.

HILLMANN, K.-H. (1981): Umweltkrise und Wertwandel. Die Umwertung der Werte als Strategie des Überlebens. - Frankfurt a.M. und Bern (= Europäische Hochschulschriften, Reihe XXII, Soziologie, Bd. 51).

LITTLE, B. R. (1983): Personal Projects. A Rationale and Method for Investigation. - In: Environment and Behavior, 15, S. 273-309.

LOCKER, A. und N. A. COULTER, Jr. (1977): A New Look at the Description and Prescription of Systems. - In: Behavioral Science, 22, S. 197-206.

LUHMANN, N. (1985): Soziale Systeme. Grundriß einer allgemeinen Theorie. - Frankfurt a. M., 2. Aufl.

MEHAN, H. und H. WOOD (1979/1975): Fünf Merkmale der Realität. - In: E. WEINGARTEN, F. SACK und J. SCHENKEIN (Hrsg.), Ethnomethodologie. Beiträge zu einer Soziologie des Alltagshandelns. - Frankfurt a. M., S. 29-63.

PACIONE, M. (1982): The Use of Objective and Subjective Measures of Life Quality in Human Geography. - In: Progress in Human Geography, 6, S. 495-514.

REICHERT, D. (1985): The Most Secure Place Is a Prisonhouse. On the Logic and the Function of Human Geography. - In: G. BAHRENBERG und J. DEITERS (Hrsg.), Zur Methodologie und Methodik der Regionalforschung. - Osnabrück, S. 19-32.

SEDLACEK, P. (Hrsg., 1982): Kultur-/Sozialgeographie. Beiträge zu ihrer wissenschaftstheoretischen Grundlegung. Paderborn u. a. (= UTB 1053).

SEDLACEK, P. (1982): Kulturgeographie als normative Handlungswissenschaft. - In: P. SEDLACEK, Hrsg., S. 187-216.

WALMSLEY, D. J. (1982): Personality and Regional Preference Structures: A Study of Introversion-Extraversion. - In: The Professional Geographer, 34, S. 279-288.

WEICHHART, P. (1986a, in Druck): Diskussionsbemerkungen zur Konzeption und zum Programm einer "geographischen Humanökologie». - In: D. STEINER und B. WISNER (Hrsg.), Geographie und Humanökologie, Zürich.

WEICHHART, P. (1986b, in Druck): Wohnsitzpräferenzen im Raum Salzburg. - Salzburg (= Salzburger Geographische Arbeiten, $\mathrm{Bd}$. 15).

WERLEN, B. (1983): Methodologische Probleme handlungstheoretischer Stadtforschung. - In: Jahrbuch der Schweizerischen Naturforschenden Gesellschaft, 1982, Bd. 2, S. 100-109.

WERLEN, B. (1986): Handlungstheoretische Sozialgeographie. - Freiburg, Diss., $22+506 \mathrm{~S}$.

WIRTH, E. (1977): Die deutsche Sozialgeographie in ihrer theoretischen Konzeption und ihrem Verhältnis zu Soziologie und Geographie des Menschen. - In: Geographische Zeitschrift, 65, S. 161-187.

WIRTH, E. (1981): Kritische Anmerkungen zu den wahrnehmungszentrierten Forschungsansätzen in der Geographie. Umweltpsychologisch fundierter "behavioural approach" oder Sozialgeographie auf der Basis moderner Handlungstheorien? - In: Geographische Zeitschrift, 69, S. 161-198.

WRIGHT, G. H. v. (1972): The Logic of Preference Reconsidered. - In: Theory and Decision, 3, S. 140-169. 